\title{
Incontinência fecal: perfil dos idosos residentes na cidade de Rio Piracicaba, MG
}

\author{
Fecd incontinence profileof ddresidentsinthecity of RioPiradicaba, \\ stateof MnesGerais, Brazil
}

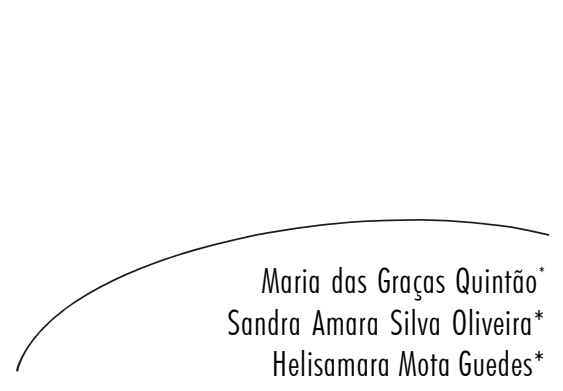

Resumo

\begin{abstract}
A incontinência fecal é uma patologia que atinge uma parcela significativa da população, principalmente os idosos. O objetivo deste estudo foi analisar os possíveis fatores que levaram ao desenvolvimento da incontinência fecal em idosos do município de Rio Piracicaba, MG. Tratou-se de pesquisa quantitativa do tipo descritiva, realizada em 2009 com 23 idosos, mediante aplicação dos questionários sócio-demográficos, seguindo o Fecal Incontinence Severity Index - FISI e da avaliação do Mini-exame do Estado Mental. Verificou-se que 52,10\% são do gênero feminino; $52,20 \%$ têm mais de 80 anos; 73,90\% são analfabetos; $95,65 \%$ têm renda mensal de um salário mínimo; $73,90 \%$ têm menos de três problemas associados; $73,90 \%$ apresentam a incontinência por um período de até 60 meses e 95,65\% não fazem tratamento para incontinência fecal. Encontrou-se uma associação relevante entre gênero, causa da doença e problemas associados ao acidente vascular encefálico. Os dados mostraram a necessidade de implementação de ações educativas visando propiciar informações sobre a doença, tratamento e sua prevenção.
\end{abstract}

\section{Abstract}

The fecal incontinence is a condition that affects a significant portion of the population, especially old people. This study aimed to examine the possible factors that caused the development of fecal incontinence in old people of the city of Rio Piracicaba, State of Minas Gerais, Brazil. This was a descriptive type of quantitative research, conducted in 2009 with 23 people, with the application of sociodemographic questionnaires, according to the Fecal Incontinence Severity Index FISI and by evaluation of the Mini-mental State Examination. It was found that $52,10 \%$ are female; $52,10 \%$ are over 80 years old; $73,90 \%$ are illiterate; $95,65 \%$ have a monthly income of one minimum wage; $73,90 \%$ have less than three associated problems; $73,90 \%$ are presenting incontinence for up to 60 months and $95,65 \%$ don't receive any treatment for this disease. It was also found a significant association between gender, disease's cause and problems associated with brain stroke. The data demonstrated the need for implementation of educational procedures to provide information about the disease, its treatment and prevention.

Centro Universitário do Leste de Minas Gerais. Curso de Enfermagem. Ipatinga, MG, Brasil.

\section{Palavras-chave:} Incontinência Fecal. Idoso. Canal Anal. Identidade de Gênero. Análise Quantitativa. Epidemiologia Descritiva. Rio Piracicaba, MG.

Key words: Fecal Incontinence. Aged. Anal Canal. Gender Identity. Quantitative Analysis. Epidemiology, Descriptive. Rio Piracicaba city. Old People. 


\section{INTRODUÇÃO}

A incontinência fecal é uma alteração funcional que leva à perda involuntária das fezes líquidas, pastosas, sólidas ou flatos, dependendo da gravidade do caso, em tempo e/ou locais inadequados. A incontinência pode gerar insegurança, perda da autoestima, angústia, depressão, transtornos físicos, mentais e sociais, que podem contribuir para uma piora na qualidade de vida dos indivíduos. ${ }^{1}$

Embora os dados sobre a prevalência da incontinência fecal sejam desconhecidos, estimase que cerca de 2 a $7 \%$ da população geral apresente algum grau de incontinência fecal, sendo esta uma condição sub-relatada, pois muitas pessoas acometidas pela incontinência não procuram assistência aos profissionais da saúde, devido ao medo, frustração e vergonha. ${ }^{2,3}$

A incontinência é a segunda causa mais comum de institucionalização. Sua ocorrência predomina entre idosos, mas as mulheres parecem ser mais suscetíveis, devido às lesões traumáticas relacionadas ao parto. ${ }^{4}$

Barbosa \& Marques, ${ }^{5}$ ao pesquisarem 39 idosos com incontinência fecal, observaram que os indivíduos tinham em média 71,92 anos, sendo a maioria do gênero feminino $(89,74 \%)$ e apenas $48,72 \%$ dos participantes já havia feito tratamento para esta condição.

A ação integrada da musculatura esfincteriana anal e dos músculos do assoalho pélvico, aliada à presença do reflexo inibitório reto anal, $\mathrm{da}$ consistência das fezes e do tempo de trânsito intestinal, são fatores dos quais depende o funcionamento normal do intestino. Entretanto, há uma série de condições clínicas que podem afetar esses mecanismos, tais como diarreias, diabetes, doenças autoimunes, síndrome do cólon irritável, doenças inflamatórias intestinais, proctite de radiação, entre outros, que podem gerar incontinência. Além disso, é possível observar, nos idosos, situações de impactação fecal, fator que pode causar laceração do músculo e incontinência por extravasamento. ${ }^{4}$
A incontinência é uma condição grave tanto para a pessoa acometida pela doença, quanto para seus cuidadores, o que reforça a importância de receberem orientação adequada a respeito das medidas que podem ser tomadas visando a um melhor controle, como por exemplo: evitar o uso de laxantes - o que pode induzir diarreia, explicar que não há necessidade de evacuar diariamente, monitorar o padrão da incontinência, a fim de determinar se ela ocorre em horário semelhantes a cada dia, recomendar o uso de roupas íntimas e absorventes higiênicos para proteger o vestuário e a pele do indivíduo. ${ }^{6,7}$

Este estudo se torna relevante por fornecer subsídios que irão contribuir para uma intervenção mais efetiva para os profissionais de saúde, a fim de reduzir o custo financeiro com a incontinência, o número de internações hospitalares e melhorar a assistência prestada ao idoso. Assim, será possível promover uma melhor qualidade de vida, evitandose inclusive problemas psicológicos que podem levar a uma reclusão social.

O presente estudo tem como objetivo caracterizar idosos com incontinência fecal quanto aos dados demográficos, sociais, clínicos e sobretudo analisar os possíveis fatores que levaram os idosos a desenvolverem a incontinência fecal no município de Rio Piracicaba-MG.

\section{METODOLOGIA}

Tratou-se de uma pesquisa com abordagem quantitativa do tipo descritivo, desenvolvido em idosos com incontinência fecal.

Esta pesquisa foi realizada em domicílios situados no interior de Minas Gerais, na cidade de Rio Piracicaba, que, de acordo com o Instituto Brasileiro de Geografia e Estatística, ${ }^{8}$ possui uma população de 14.326 habitantes. Foram incluídos como sujeitos de pesquisa indivíduos com idade igual ou maior a 60 anos, dos gêneros masculino e feminino, com sintoma de incontinência fecal. Ressalta-se que se adotou como critério de exclusão da pesquisa a recusa em participar do estudo. 
Para este estudo, foi considerada uma população constituída por 56 idosos cadastrados na distribuição de fraldas geriátricas da Unidade Básica de Saúde (UBS) do referido município, sendo que a amostra foi composta por 23 idosos, já que os registros da UBS estavam desatualizados, ocorreram sete óbitos e três haviam mudado de endereço. Foram excluídos 23 indivíduos, por apresentaram incontinência urinária.

Como instrumento de coleta de dados, foi utilizado um questionário Clínico-SócioDemográfico, ${ }^{9}$ que continha informações pessoais do participante, problemas de saúde, ocorrências proctológicas, ginecológicas e urológicas - o Fecal Incontinence Severity Index (FISI) ${ }^{9}-$, para caracterizar a gravidade de incontinência fecal, e o Mini-Exame do Estado Mental (MEEM), ${ }^{9}$ para analisar as habilidades linguísticas dos idosos e sua maneira de processar informações, quanto à orientação no tempo e espaço, sendo necessário para responder os questionários aplicados.

Os parâmetros de referência utilizados para o teste do Mini-Exame do Estado Mental foram: 30-26 pontos (funções cognitivas preservadas), de 26-24 pontos (alterações não sugestivas de déficit), 23 pontos ou menos (sugestivo de déficit cognitivo). ${ }^{10}$

Para a coleta de dados, foram extraídas informações do cadastro de distribuição de fraldas, sendo agendado pelas funcionárias da UBS o encontro das pesquisadoras com os possíveis participantes da pesquisa. Antes de aplicar os questionários, realizou-se levantamento prévio com os idosos sobre a dificuldade de controlar o intestino. $\mathrm{O}$ idoso que não apresentou condições de comunicação verbal para responder aos instrumentos de coleta de dados teve o questionário respondido por seu cuidador ou responsável. A ordem de aplicação dos questionários foi: Mini-Exame do Estado Mental, Clínico-Sócio-Demográfico e Fecal Incontinence Severity Index.

A coleta de dados foi realizada no período de novembro de 2008 a janeiro de 2009 , com tempo de aproximadamente 40 minutos para cada participante. Todos os questionários foram aplicados em um mesmo encontro, nos domicílios dos participantes. Os dados coletados foram demonstrados através de tabelas e comparados com a literatura referente ao tema.

A pesquisa foi aprovada pelo Comitê de Ética do Centro Universitário do Leste de Minas Gerais - UnilesteMG, protocolo $\mathrm{n}^{\mathrm{o}}$ 17.95.08, e autorizada pela secretaria de saúde do referido município. Foi realizada dentro dos padrões éticos, garantindo a todos os participantes sigilo e anonimato. Solicitou-se a assinatura do Termo de Consentimento Livre e Esclarecido em duas vias de igual teor, ficando uma com o entrevistado e outra com as pesquisadoras. A pesquisa contemplou a Resolução no 196/1996, do Conselho Nacional de Saúde, que regulamenta a pesquisa com seres humanos. ${ }^{11}$

Aos participantes, foi oferecida gratuitamente uma cartilha com o tema da incontinência fecal, contendo informações globais sobre a doença e orientações para facilitar o seu controle.

\section{RESULTADOS}

Participaram do estudo 23 idosos com incontinência fecal, cujas características sóciodemográficas podem ser visualizadas na tabela 1 . 
Tabela 1 - Informações descritivas sobre as características sócio - demográficas da amostra $(n=23)$. Ipatinga, MG, 2009.

\begin{tabular}{lcc}
\hline \multicolumn{1}{c}{ Variável } & $\mathrm{N}$ & $\%$ \\
\hline Gênero & 12 & $52,10 \%$ \\
Feminino & 11 & $47,90 \%$ \\
Masculino & & \\
Idade (anos) & 3 & $13,15 \%$ \\
$60-69$ & 8 & $34,75 \%$ \\
$70-79$ & 12 & $52,10 \%$ \\
Mais de 80 anos & & \\
Estado civil & 13 & $56,55 \%$ \\
Viúvo & 6 & $26,00 \%$ \\
Solteiro & 4 & $17,45 \%$ \\
Casado & & \\
Escolaridade & 17 & $73,90 \%$ \\
Analfabetos & 5 & $21,75 \%$ \\
Ensino fundamental - até 7 anos & 1 & $4,35 \%$ \\
Ensino Médio -mais de 8 anos & & \\
Arranjo familiar (no e categorias de pessoas com & & \\
quem reside) & 12 & $52,10 \%$ \\
Filhos & 7 & $30,45 \%$ \\
Outros (sobrinhos e irmãos) & 4 & $17,45 \%$ \\
Cônjuge & & \\
Renda Individual & 22 & $95,65 \%$ \\
1 salário mínimo & 1 & $4,35 \%$ \\
Sem rendimento & & \\
Ocupação & 23 & \\
Aposentado & & \\
\hline
\end{tabular}

Este estudo foi constituído, em sua maioria, por sujeitos do gênero feminino- 12 mulheres $(52,10 \%)$. Predominou em $52,10 \%$ a faixa etária de mais de 80 anos. Em relação ao estado civil, $13(56,55 \%)$ eram viúvos. No que se refere à escolaridade, 17 (73,90\%) eram analfabetos e quanto ao arranjo familiar, quatro $(17,45 \%)$ residiam com cônjuge e $19(82,60 \%)$ com filhos e/ ou agregados (sobrinhos, irmãos). Vinte e dois $(95,65 \%)$ entrevistados apresentaram renda individual de um salário mínimo. Quanto à ocupação, 23 (100\%) eram aposentados.
As informações clínicas dos idosos encontraram-se na tabela 2. Sob o aspecto clínico, 17 idosos $(73,90 \%)$ informaram ter menos de três patologias associadas, sendo as mais frequentes: acidente vascular encefálico, diabetes e hipertensão. Com relação ao número de medicamentos, nove $(39,10 \%)$ consumiam de três a cinco drogas. A maioria da amostra, 22 indivíduos (95,65\%), informou não praticar nenhuma atividade física. 
Tabela 2 - Informações descritivas sobre as variáveis pesquisadas na avaliação clínica da amostra ( $\mathrm{n}=$ 23). Ipatinga, MG, 2009.

\begin{tabular}{lcc}
\hline \multicolumn{1}{c}{ Variável } & $\mathrm{N}$ & $\%$ \\
\hline Número de problemas de saúde associados & & \\
Menos de 3 & 17 & $73,90 \%$ \\
de 4-6 & 5 & $21,75 \%$ \\
Mais de 7 & 1 & $4,35 \%$ \\
Número de medicamentos & & \\
Nenhum & & \\
1 a 2 & 2 & $8,70 \%$ \\
3 a 5 & 7 & $30,45 \%$ \\
maior que 5 & 9 & $39,10 \%$ \\
& 5 & $21,75 \%$ \\
Atividade Física & & \\
Não & & \\
Sim & 22 & $95,65 \%$ \\
\hline
\end{tabular}

A maioria das mulheres, $10(83,30 \%)$, informou ter tido mais de sete gestações com partos vaginais e apenas duas (16,70\%) eram nulíparas. Entre os homens, dois $(18,18 \%)$ tinham tumor de próstata. Na tabela 3, podem ser visualizadas as características da incontinência fecal e os sintomas proctológicos dos participantes.

Tabela 3 - Informações descritas relativas à caracterização da incontinência fecal e dos sintomas proctológicos $(n=23)$. Ipatinga, MG, 2009.

\begin{tabular}{|c|c|c|}
\hline Variável & $\mathrm{N}$ & $\%$ \\
\hline \multicolumn{3}{|l|}{ Sintomas proctológicos } \\
\hline Diarreia & 6 & $26,00 \%$ \\
\hline Hemorróidas & 2 & $8,70 \%$ \\
\hline Impactaçao fecal & 2 & $8,70 \%$ \\
\hline Outros (retocele) & 2 & $8,70 \%$ \\
\hline Constipação intestinal & 1 & $4,35 \%$ \\
\hline Não possui & 10 & $43,55 \%$ \\
\hline \multicolumn{3}{|l|}{ Incontinência urinária } \\
\hline Não & 12 & $52,10 \%$ \\
\hline $\operatorname{Sim}$ & 11 & $47,90 \%$ \\
\hline \multicolumn{3}{|l|}{ Tempo de incontinência fecal (meses) } \\
\hline Até 2 meses & 4 & $17,45 \%$ \\
\hline 13 a 60 meses & 13 & $56,55 \%$ \\
\hline Mais de 60 meses & 6 & $26,00 \%$ \\
\hline
\end{tabular}


Tabela 3 - Informações descritas relativas à caracterização da incontinência fecal e dos sintomas proctológicos $(n=23)$. Ipatinga, MG, 2009. (continuação)

\begin{tabular}{lcc}
\hline \multicolumn{1}{c}{ Variável } & $\mathrm{N}$ & $\%$ \\
\hline Circunstâncias da incontinência fecal & & \\
Não percebe & 13 & $56,55 \%$ \\
Percebe, mas não retém & 7 & $30,45 \%$ \\
Duas ou mais circunstancias associadas & 3 & $13,00 \%$ \\
& & \\
Uso de proteção & 23 & $100 \%$ \\
Fralda geriátrica & & \\
Tratamento para incontinência fecal & & \\
Não & 22 & $95,65 \%$ \\
Sim & & $4,35 \%$ \\
& & \\
Número de evacuações diárias & & $60,90 \%$ \\
1 - 2 & 14 & $39,10 \%$ \\
$3-4$ & 9 & \\
Consistência das fezes & & $34,75 \%$ \\
Líquidas & & $26,00 \%$ \\
Variável & 8 & $17,45 \%$ \\
Sólidas & 6 & $17,45 \%$ \\
Pastosas & $6,35 \%$ \\
Ressecadas & 4 & \\
\hline & 4 & 1 \\
\hline
\end{tabular}

Em relação à sintomatologia proctológica relacionada à incontinência fecal, seis $(26,00 \%)$ apresentaram sintomas como diarreia e 10 $(43,55 \%)$ não apresentaram sintomas.

Da amostra total, $11(47,90 \%)$ idosos relataram, além de incontinência fecal, queixa de incontinência urinária. Com relação ao tempo de incontinência fecal, 13 (56,55\%) apresentaram sintomas de 13 a 60 meses.

A maioria dos entrevistados, 13 (56,55\%), apresentou como circunstâncias da incontinência a não-percepção da presença das fezes.

Quanto ao uso de proteção, 23 (100\%) idosos usavam fraldas geriátricas e como complementação, panos, e $22(95,65 \%)$ não faziam tratamento para a incontinência fecal. Quanto ao número de evacuações, 14 (60,90\%) evacuavam até duas vezes por dia e nove $(39,10 \%)$, mais de três evacuações diárias.

Quando se analisou a consistência das fezes, foi observada predominância significativa de fezes líquidas em oito indivíduos (34,75\%). Em relação ao hábito intestinal, 18 (78,23\%) da amostra relataram ter flatulência diária.

Para analisar a habilidade linguística dos idosos, foi utilizado o Mini-Exame do Estado Mental, identificando que dos 23 participantes da pesquisa, somente $10(43,47 \%)$ idosos apresentaram condições físicas e mentais para realizar o teste, sendo que nove (90\%) apresentaram valores sugestivos de déficit cognitivo e um (10\%) não apresentou déficit de cognição. A tabela 4 mostra a gravidade da incontinência fecal de acordo com Fecal Incontinence Severity Index - FISI. 
Tabela 4 - Fecal Incontinence Severity Index - FISI.

\begin{tabular}{lcccccc}
\hline $\begin{array}{l}\text { Tipo de } \\
\text { incontinência }\end{array}$ & Nunca & $\begin{array}{l}\text { 1 a 3 vezes } \\
\text { por mês }\end{array}$ & $\begin{array}{l}1 \text { vez por } \\
\text { semana }\end{array}$ & $\begin{array}{c}\text { 2 ou mais } \\
\text { vezes por } \\
\text { semana }\end{array}$ & $\begin{array}{l}1 \text { vez por } \\
\text { dia }\end{array}$ & $\begin{array}{l}2 \text { ou mais } \\
\text { vezes por }\end{array}$ \\
\hline Flatos (gases) & 0 & 2 & 1 & 2 & 7 & dia \\
Muco & 21 & 1 & 0 & 1 & 0 & 0 \\
Fezes líquidas & 0 & 1 & 4 & 3 & 2 & 13 \\
Fezes sólidas & 0 & 2 & 9 & 3 & 4 & 5 \\
\hline
\end{tabular}

Em relação ao tipo de incontinência fecal e frequência, 11 (47,90\%) indivíduos relataram que eliminam flatos duas ou mais vezes por dia, 21 $(91,30 \%)$ nunca tiveram muco nas fezes, 13 $(56,55 \%)$ apresentaram ter fezes líquidas duas ou mais vezes por dia e nove $(39,10 \%)$ evacuavam uma vez por semana, com características sólidas.

\section{DISCUSSÃO}

O estudo mostrou que a incontinência fecal é predominante em mulheres, $(52,10 \%)$, e idosas acima de 80 anos.

Barbosa $^{9}$ avaliou a qualidade de vida e as estratégias de enfrentamento em 39 idosos com incontinência fecal, encontrando que $89,74 \%$ dos participantes eram do gênero feminino e $46,15 \%$ apresentaram a faixa etária entre 70-79 anos, dados superiores ao encontrado neste estudo.

Percebeu-se maior predomínio $\mathrm{da}$ incontinência fecal em idosas, o que pode ser devido a fatores relacionados ao parto e à expectativa de vida das mulheres, que é maior que a dos homens. ${ }^{4}$

Há, no Brasil, uma proporção de 78,6 homens idosos para cada 100 mulheres idosas, considerados acima de 60 anos. Nessa faixa etária, o diferencial por gênero, acentuado nos últimos anos, pode ser explicado pela diferença no ritmo de crescimento desta parcela da população. ${ }^{12}$

O presente estudo mostrou que $53,55 \%$ idosos eram viúvos e $82,60 \%$ residiam com filhos e/ou agregados (sobrinhos, irmãos). Leite et al. ${ }^{13}$ analisaram o suporte familiar e social dos 338 idosos que residem no meio urbano de AlecrinRS, encontrando que $61,5 \%$ eram casados e $49,7 \%$ dos idosos residiam com seu cônjuge.

De acordo o Censo 2000, há uma proporção de cinco mulheres viúvas para cada homem viúvo, representados por 1.149.157 homens viúvos e 5.082.116 mulheres nesta condição. ${ }^{14}$ Tal situação ocorre devido ao fato de as mulheres se casarem mais jovens que seus maridos, além de possuírem maior longevidade e dificilmente se casarem novamente. $^{15}$

Quanto à escolaridade, 73,90\% eram analfabetos, 95,65\% apresentaram renda até um salário mínimo e 23 (100\%) dos indivíduos eram aposentados.

Campos et al. ${ }^{16}$ analisaram a distribuição espacial dos idosos segundo características demográficas, sociais e de morbidade mais prevalente dos 468 idosos do município de Botucatu-SP, encontrando que $20 \%$ dos idosos entrevistados não frequentaram escola e 50\% possuíam apenas até o $1^{\circ}$ grau completo; $42,5 \%$ recebiam rendimento de até três salários mínimos, originados de aposentadorias e pensões. No presente estudo, a quantidade de idosos analfabetos foi maior do que nos estudos citados.

O baixo nível de escolaridade dos idosos brasileiros reflete a desigualdade social e as políticas de educação predominantes nas décadas de 1930 e 1940, nas quais o acesso à escola era muito 
limitado. Ao considerar o número médio de anos de estudo dos idosos responsáveis pelos domicílios no Brasil no ano 2000, o Censo Demográfico aponta para apenas 3,4 anos, sendo 3,5 anos para os homens e 3,1 anos de estudo para as mulheres. ${ }^{16}$

Este estudo mostra alguns dos aspectos clínicos dos idosos, sendo que $73,90 \%$ informaram ter menos de três patologias associadas, sendo as mais frequentes: acidente vascular encefálico, diabetes e hipertensão. Com relação ao número de medicamentos, $39,10 \%$ consumiam de três a cinco drogas. A maioria, 95,65\%, informou não praticar nenhuma atividade física.

Barbosa $^{9}$ demonstrou que $53,85 \%$ informaram ter de quatro a seis problemas de saúde associados; $41,02 \%$ informaram utilizar de três a cinco medicamentos e $66,67 \%$ informaram realizar atividade física.

O avanço da idade é um fator que aumenta as chances de ocorrência de doenças e de prejuízos à funcionalidade física, psíquica e social. Nessa etapa da vida, o aparecimento de duas ou mais doenças é bastante comum. ${ }^{10}$

Percebeu-se, neste estudo, que $47,90 \%$ dos entrevistados apresentaram incontinência urinária e 26,00\% apresentaram sintomas como diarreia. Esse dado é inferior ao estudo de Barbosa, ${ }^{9}$ segundo o qual $69,23 \%$ dos idosos referiram ter incontinência urinária e 46,15\% apresentaram diarreia.

As perdas urinária e fecal são os distúrbios mais comumente encontrados no padrão de eliminação. Tais problemas podem ser causados por alterações da função dos sistemas geniturinário ou gastrointestinal, associados à idade ou a uma doença de menor gravidade, ou ainda devido à anormalidade de outros sistemas do organismo, como o sistema nervoso e o músculo-esquelético. A associação $\mathrm{da}$ incontinência urinária e fecal pode ocorrer devido à restrição da mobilidade. ${ }^{17}$

Verificou-se que $56,55 \%$ idosos apresentavam sintomas de incontinência de 13 a 60 meses, e
$95,65 \%$ dos indivíduos não faziam tratamento para incontinência fecal.

Santos e Silva ${ }^{18}$ avaliaram a qualidade de vida de 41 pessoas portadoras de incontinência anal da cidade de São Paulo, encontrando que no tempo de sintomatologia predominam os períodos mais longos, acima de cinco anos $(46,4 \%)$. Segundo os autores, todos já haviam se submetido a tratamento prévio, a maioria $(56,09 \%)$ do tipo clínico, sendo que tais fatores caracterizam a cronicidade da incontinência fecal e a possibilidade do maior comprometimento de sua qualidade de vida.

O tratamento para incontinência fecal se dá de acordo com sua etiologia, podendo ser cirúrgico, não-cirúrgico e medicamentoso. ${ }^{18} \mathrm{O}$ tratamento cirúrgico inclui reconstrução cirúrgica, reparação do esfíncter ou desvio fecal e o não-cirúrgico é a terapia biofeedback, que inclui um treinamento de reeducação dos músculos do assoalho pélvico para aumentar o limiar de sensibilidade da distensão retal e melhorar a contratilidade do esfíncter anal. ${ }^{19} \mathrm{O}$ tratamento medicamentoso é realizado com antidiarreicos opiódes, como por exemplo, a loperamida, que é um medicamento paliativo. ${ }^{20}$

O presente estudo mostrou que dos 23 indivíduos estudados $56,55 \%$ não têm percepção da perda das fezes e apenas $30,45 \%$ a percebem, mas não conseguem impedir a perda, e os 23 $(100 \%)$ apresentaram necessidade de usar fraldas geriátricas.

Baldez ${ }^{21}$ analisou os sintomas clínicos frente aos resultados da manometria anoretal em 120 indivíduos com incontinência fecal, encontrando que $58 \%$ apresentavam necessidade de usar protetores; $50 \%$ dos entrevistados não percebiam a perda das fezes e $70 \%$ tinham incapacidade de retardar uma evacuação. Como este estudo apresentou um índice de retenção de fezes menor que o estudo comparado, faz-se necessário realizar uma avaliação clínica mais criteriosa. Já em relação ao uso de fraldas geriátricas, este estudo apresentou maior prevalência devido a sequelas do acidente vascular cerebral. 
Nos resultados obtidos com a aplicação do FISI, observou-se que a diarreia e flatos foram as eliminações mais prevalentes, com frequência de duas ou mais vezes por dia. $O$ padrão normal de eliminação intestinal é bastante individualizado e a frequência de defecção pode apresentar variação de uma ou duas eliminações diárias. Entretanto, é possível que o volume das fezes seja de tal forma abundante e o estímulo para a defecação tão intenso que o indivíduo fica incapacitado de manter a contração esfincteriana por tempo suficiente para que tenha acesso às instalações sanitárias adequadas e a remoção do vestuário, caracterizando quadros de incontinência. $^{22}$

Os flatos são acúmulos de gases presentes no trato gastrointestinal. Muitos alimentos ricos em fibras, tais como cebolas, repolho e legumes, que são indicados para promover a eliminação intestinal adequada, podem provocar produção excessiva de flatos, quando não são introduzidos de maneira gradual na dieta. ${ }^{22}$

Lourenço \& $\operatorname{Veras}^{23}$ avaliaram as características de medida do MEEM em 303 idosos atendidos em um ambulatório geral, encontrando que $71,6 \%$ eram do gênero feminino, $70 \%$ tinham mais de 70 anos de idade e $46 \%$ declaram ter até quatro anos de escolaridade.

Em relação ao MEEM, pode-se afirmar que a idade e escolaridade influenciam de maneira significativa na pontuação do MEEM; portanto, os idosos que apresentaram maior idade e menor escolaridade apresentaram um valor sugestivo de déficit cognitivo, ou seja, menor pontuação.

\section{CONCLUSÃO}

Dentro dos resultados obtidos, deve-se ressaltar que a maioria dos idosos era do gênero feminino, com idade acima 80 anos, e sedentários, o que constituem fatores que provavelmente influenciaram o desenvolvimento da incontinência em idosos.

Encontrou-se associação relevante entre gênero feminino e masculino, mostrando maior predomínio da incontinência fecal em idosas. Isto pode ser devido a fatores relacionados ao parto e ao fato de as mulheres viverem mais que os homens. Outro fator encontrado foi entre os aspectos clínicos dos idosos, que informaram ter menos de três patologias associadas, sendo as mais frequentes: acidente vascular encefálico, diabetes e hipertensão, consumo de três a cinco drogas e nenhuma atividade física. Outros fatores como a renda e o nível de educação não demonstraram influenciar nos fatores que levam a incontinência.

Uma vez que o presente estudo foi desenvolvido com um grupo específico de idosos, a detecção de fatores que levaram à incontinência fecal sugere a necessidade do estabelecimento de ações de promoção de saúde e prevenção como programa de recondicionamento intestinal, estímulo à ingestão de líquidos, informações sobre a doença e necessidade de tratamento, a fim de prevenir complicações futuras. Tais ações são inerentes ao profissional de saúde, que poderá desenvolver papel imprescindível para a modificação efetiva do cenário encontrado, dando atenção especial ao tratamento (já que grande número de idosos não faz tratamento), incluindo os cuidados na manutenção de alimentação adequada e cuidados com a integridade cutânea.

O aumento da população idosa no Brasil implica a necessidade de se dispor de maior número de profissionais preparados para lidar com o envelhecimento e, consequentemente, com as doenças inerentes a esta fase da vida. 


\section{REFERENNCIAS}

1. Oliveira SCM, et al. Incontinência fecal em mulheres na pós-menopausa: prevalência, intensidade e fatores associados. [acesso $08 \mathrm{fev}$. 2009]. Arquivo de Gastroenterologia 2006 abr./ jun; 2006. 43(2): 102-6. Disponível em: URL: < http://bases.bireme.br/cgibin/wxislind.exe/ iah/online/?IsisScript = iah/ iah.xis\&src $=$ google\&base $=$ LILACS\&lang $=$ \&nextAction $=\operatorname{lnk} \&$ exprSearch $=435252$ \&indexSear $\mathrm{ch}=\mathrm{ID} />$.

2. Bezerra CA. Continência versus Incontinência: uma abordagem conceitual. Rev Esc Enferm USP 1999; 33: 91.

3. Oliveira L, et al. Novos tratamentos para a incontinência anal: injeção de silicone melhora a qualidade de vida em 35 pacientes incontinentes. [acesso 10 fev. 2009]. Revista Brasileira de Coloproctologia 2007 abr./jun; 2007. 27(2): 167-173. Disponível em: URL: < http://www.scielo.br/pdf/rbc/v27n2/06.pdf $>$.

4. Oliveira L. Incontinência fecal. [acesso 06 fev. 2009]. Jornal Brasileiro Gastrolologia 2006 jan./ mar; 6(1): 35-7. Disponível em: URL: < http:// www.socgastro.org.br/site/scripts/revistas/ jbg01/jbg106incfecal.pdf $>$.

5. Barbosa JMM, Marques LM. Incontinência Anal. In: Baracho E. Fisioterapia aplicada à Obstetrícia. Rio de Janeiro: Medsi; 2002.

6. Nettina SM. Prática de enfermagem. 7. ed. Rio de Janeiro: Guanabara Koogan;2003. 553 p.

7. Timby BK. Conceitos e habilidades fundamentais no atendimento de enfermagem. 8. ed. Porto Alegre: Artmed; 2007. 911 p.

8. IBGE. Contagem da População 2007 e Estimativas da População 2007. [acesso 12 jan. 2009]. Rio de Janeiro: IBGE, 2007. Disponível em: URL: < http://www.ibge.gov.br/home/esta tistica/populacao/ contagem2007/MG.pdf > .

9. Barbosa JMM. Avaliação da qualidade de vida e das estratégias de enfrentamento em idosos com incontinência fecal. [dissertação]. Belo Horizonte: Universidade Federal de Minas Gerais, Ciências de Reabilitação; 2006.

10. Freitas EV, et al. Tratado de Geriatria e Gerontologia. Rio de Janeiro: Guanabara Koogan, 2002; 1187 p.

11. Brasil. Conselho Nacional de Saúde. Resolução $\mathrm{n}^{\mathrm{o}}$ 196. Diretrizes e Normas Regulamentadoras sobre Pesquisa Envolvendo Seres Humanos 1996 out. Disponível em: URL: < http:// www.unb.br/fs/clm/labcor/etic196.htm - 65k >.
12. IBGE. Perfil dos idosos responsáveis pelos domicílios no Brasil: 2000. [acesso 04 mar. 2009]. Rio de Janeiro: IBGE, 2006. Disponível em: URL: < http://www.ibge. gov.br/home/ estatistica/ populacao/perfilidoso/ default.shtm $>$.

13. Leite MT, et al. Idosos residentes no meio urbano e sua rede de suporte familiar e social. [acesso 26 jan. 2009]. Texto \& Contexto de Enfermagem 2008 abr./jun; 17(2): 250-7. Disponível em: URL: < http://www.scielo.br/ pdf/tce/v17n2/05.pdf $>$.

14. IBGE. Censo demográfico 2000: agregado por setores censitários dos resultados do universo: Região Sul. [acesso 26 fev. 2009]. Rio de Janeiro: IBGE, 2002. Disponível em: URL: < http:// www.ibge.gov. br/lojavirtual/ lojavirtual.php? midia $=\mathrm{DI}-31 \mathrm{k}>$.

15. Buaes CS, Doll J. Aprende a ser viúva: narrativas de mulheres idosas no meio rural. [acesso 19 abr. 2009]. Revista Kairós; 2005; 8(2): 171-88. Disponível em: URL: < http:// bases.bireme.br/cgi-bin/wxislind.exe/iah/ online/? IsisScript $=$ iah/ iah. $x$ is\&src $=$ google\&base $=$ LILACS\&lang $=$ p\&nextAction $=\operatorname{lnk} \&$ exprSearch $=$ 484033\&indexSearch $=$ ID $>$.

16. Campos FG, et al. Distribuição espacial dos idosos de um município de médio porte do interior paulista segundo algumas características sócio-demográficas e de morbidade. [acesso 23 fev. 2009]. Cad Saúde Pública 2009 jan; 25(1): 7786. Disponível em: URL: < http:// www.scielo.br/pdf/csp/v25n1/08.pdf $>$.

17. Felten BS, et al. Geriatria e Gerontologia. Rio de Janeiro: Reichmann e Autores Editores; 2005--.-. $352 \mathrm{p}$.

18. Santos VLCG, Silva AM. Qualidade de vida em pessoas com incontinência anal. [acesso 21 jan. 2009]. Revista Brasileira de Coloproctologia 2002; 22(2): 98-108. Disponível em: URL: < http://www.sbcp.org.br/revista/nbr222/ P98_108. htm - 82k $>$.

19. Galvão-Alves J, Dani R. Terapêutica em Gastroenterologia. Rio de Janeiro; Guanabara Koogan; 2005. 174 p.

20. Smeltzer SC, Bare BG. Tratado de Enfermagem Médico- Cirúrgico. 10. ed. Rio de Janeiro: Guanabara Koogan; 2005. 679 p.

21. Baldez JR. Relação entre os sintomas clínicos da incontinência anal e os resultados da manometria 
anoretal. [acesso 10 fev. 2009]. Revista Brasileira de Coloproctologia 2004 abr./jun; 24(2): 140-143.

Disponível em: URL: < http://www.sbcp.org.br/ revista/nbr242/P140_143.htm >.

22. Craven RF, Hirnle CJ. Fundamentos de

Enfermagem: saúde e função humana. 4. ed. Rio de Janeiro: Guanabara Koogan; 2006. 1586 p.
23. Lourenço RA, Veras RP. Mini-Exame do Estado Mental: características psicométricas em idosos ambulatoriais. [acesso 13 fev. 2009]. Rev Saúde Pública 2006 ago; 40(4): 712-9. Disponível em:

URL: < http://www.scielosp.org/

scielo.php?pid $=$ S003489102006000500023

\&script $=$ sci_arttext $-61 \mathrm{k}>$.

Recebido: 20/7/2009

Aprovado: 01/12/2009 
